\title{
Catalytic Asymmetric Synthesis of Apple Leafminer Pheromones
}
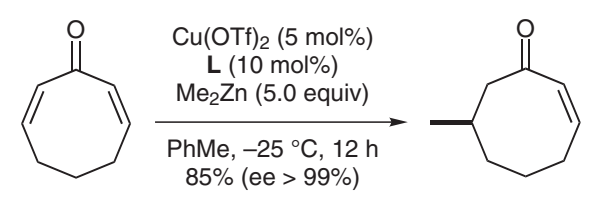

A
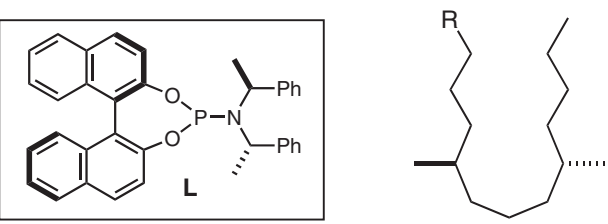

C R $=\left(\mathrm{CH}_{2}\right)_{4} \mathrm{CH}=\mathrm{CH}_{2}$ D R $=\left(\mathrm{CH}_{2}\right)_{4} \mathrm{CH}_{3}$



ee $>99 \%$, de $>98 \%$

TMSOTf (5.0 equiv) r.t., $2 \mathrm{~h}$

Synthesis of

Natural Products and Drugs

Key Words

enantioselective catalytic 1,4addition

desymmetrization

catalyst control

remote

stereocontrol

organozinc

reagents

phosphoramidite

Significance: Starting from cycloocta-2,7-dienone, all four diastereoisomeric 8-hydroxy-3,7dimethyloctanoic acids were prepared in four steps (38\% overall) and one of them (B) was transformed to pheromones $\mathbf{C}$ and $\mathbf{D}$ of the apple leafminer (Lyonetia prunifoliella) in a further four steps. A new method for the desymmetrization of crossconjugated dienones is presented.
Comment: The key step in the synthesis is the powerful Cu-phosphoramidite-catalyzed enantioselective conjugate addition of dialkylzincs to enones. The method offers a general solution to the construction of carbon chains with alkyl groups in a 1,5-relationship. It complements the procedures of Noyori (Ru-catalyzed asymmetric hydrogenation of allylic alcohols) and Negishi (Zrcatalyzed enantioselective carboalumination) for the synthesis of isoprenoid chains. For reviews of enantioselective $\mathrm{Cu}$-catalyzed conjugate addition see: A. Alexakis, C. Benhaim Eur. J. Org. Chem. 2002, 3221-3236; B. L. Feringa Acc. Chem. Res. 2000, 33, 346-353. 